\title{
Single/Multi-User MIMO Differential Capacity
}

\author{
Daniel Castanheira and Atílio Gameiro \\ University of Aveiro \\ (Instituto de Telecomunicações) \\ Portugal
}

\section{Introduction}

This chapter will be structured around two contributions by the authors on the topic, (Castanheira \& Gameiro 2008) and (Castanheira \& Gameiro 2009).

The provision of broadband services to everyone is considered one of the key components for enabling the so-called information society. It is more or less consensual that to attain the high-rates envisioned by IMT-2000 (I. R. R. M. M1645 2003) of providing around 1Gbit/s for pedestrian and $100 \mathrm{Mbit} / \mathrm{s}$ for high mobility, will require the use of multiple antennas at the transceivers, to exploit the scattering properties of the wireless medium. Nevertheless, due to the physical size limitations of the transceivers, the number of antennas cannot be high and the space between them is limited, which implies that the degree of channel independence achieved is not sufficient to attain the high capacities envisioned, in most scenarios. One possible solution to cope with this problem is to have the mobiles simultaneously communicating with a group of geographically distributed antennas with perfect cooperation between them. The key to achieve perfect cooperation is to have the radio signals transparently transmitted / received to / from a central unit (CU), where all the signal processing is done (FUTON 2008). Considering the high capacities envisioned optical fiber, due to its low attenuation and enormous bandwidth is the obvious technology of choice to build these transparent interconnections. However the joint processing of a group of antennas and the remote transmission of their signals to the $\mathrm{CU}$ will require additional processing power and will imply additional costs to the overall network. It is also expected, by the law of diminishing returns, that as more and more antennas are jointly processed the improvement in throughput will not increase linearly with the added complexity. Thus a tradeoff must be made between the costs/complexity and the number of antennas deployed. One possible way to ease the complexity problem is to use low complexity/sub-optimal schemes, like Zero-Forcing (ZF) (Caire \& Shamai 2003) or BlockDiagonalization (BD) (Seijoon Shim et al. 2008) at the CU. Following this line of thought, in (Jindal 2005) and (Juyul Lee \& Jindal 2007), the authors study the incurred losses, in terms of power/rate offsets, between ZF/BD and the optimal scheme, which is well known to be Dirty Paper Coding (DPC). The authors conclude that the losses are higher when the number of transmit antennas is close to the number of aggregate receive antennas. Thereafter the analysis of the gains introduced by the connection of the system users to more transmit antennas is of special importance, either to estimate a "reasonable" number of 
antennas that should be jointly processed, either to give a measure for the network to check if, in a distributed antenna system (DAS), it is worthwhile to provide an additional connection to the mobiles, or to provide some guidelines for the deployment of the distributed antennas or even to compare sub-optimal schemes to the optimal scheme. In that context we define Differential CAPacity (DCAP) as the increase in ergodic sum-capacity when one additional transmit antenna is connected to the system users, to quantify the gains provided by the processing of one additional transmit antenna at the $\mathrm{CU}$ and analyze its behavior.

This chapter is organized as follows: section 2 presents the channel model; section 3 describes the single-user and multi-user channel capacities. In section 4 and 5 the DCAP, for the single-user and multi-user scenarios, is studied. In section 6 numerical results are provided and finally in section 7 some conclusions are drawn.

\section{Channel Model}

Throughout this chapter a Distributed Multiple-Input Multiple-Output (MIMO) Broadcast channel with $\mathrm{K}$ users, each with $\mathrm{N}$ receive antennas, and $\mathrm{M}$ transmit antennas is considered. A broadcast channel (BC) is a communication channel in which there is one sender and two or more receivers (Cover 1972). For a broadcast channel, the user $\mathrm{k}$ received signal, $\mathbf{y}_{k}\left(\in \mathbb{C}^{N \times 1}\right)$ can be modeled by:

$$
\mathbf{y}_{k}=\mathbf{H}_{k} \mathbf{x}+\mathbf{n}_{k}, \quad k=1, \ldots, K
$$

where $\mathbf{H}_{k}\left(\in \mathbb{C}^{N \times M}\right)$ is the user $k$ channel matrix, $\mathbf{x}\left(\in \mathbb{C}^{M \times 1}\right)$ is the transmitted signal vector with power constraint $\mathbb{E}\left[\mathbf{x x}^{H}\right] \leq P$ and $\mathbf{n}_{k}\left(\in \mathbb{C}^{N \times 1}\right)$ is complex white Gaussian noise with zero mean and unit variance per vector component $\left(\tilde{\mathcal{N}}_{N}(\mathbf{0}, \mathbf{I})\right)$. $\mathbf{H}^{H}=\left[\mathbf{H}_{1}^{H}, \ldots, \mathbf{H}_{K}^{H}\right]$ denotes the concatenation of all channels and is matrix-variate complex Gaussian distributed (Shin \& Lee 2003), with zero mean and covariance $\Sigma\left(\in \mathbb{C}^{K N M \times K N M}\right)$. Through the chapter it is assumed that the receiver has perfect knowledge of its own channel and the transmitter has perfect knowledge of all channels, for each channel realization. Since a DAS is considered all channel gains are independent $(\boldsymbol{\Sigma}$ is diagonal).

Notations: Boldface letter denote matrix-vector quantities. The operation $\operatorname{tr}(),.(.)^{H}$ and $|$. represents the trace, the Hermitian transpose and the determinant of a matrix, respectively. By $\mathbf{A}>0$ and $\mathbf{I}_{n}$ we denote that $\mathbf{A}$ is positive definite and an $n \times n$ identity matrix. The notation $\mathbf{a} \sim \tilde{\mathcal{N}}_{p}(0, \mathbf{\Sigma})$ and $\mathbf{B} \sim \tilde{\mathcal{W}}_{p}(n, \boldsymbol{\Gamma})$ is used to denote that the column vector $\mathbf{a}$ is distributed as p-variate complex Gaussian, with zero mean and covariance $\boldsymbol{\Sigma} \in \mathbb{C}^{p \times p}$, and to denote that matrix $\mathbf{B} \in \mathbb{C}^{p \times p}$ is complex Wishart distributed, with $n$ degrees of freedom and with mean $\boldsymbol{\Gamma}$. The notation $\mathbf{A} \sim \mathbf{B}$ denotes that matrix $\mathbf{A}$ and $\mathbf{B}$ are identically distributed. 


\section{Single/Multi-User Sum-Capacity}

\subsection{Single-User case}

For the single-user case, if only one receive antenna is considered, the system reduces to the classical multiple input single output (MISO) system. As a consequence, the resulting capacity expression are much simpler to analyze than for the general case of a multi-user system. Thus for this case, the system capacity and the corresponding DCAP is described in more detail, either to introduce the reader to the topic, either to provide some baseline work to be used in the multi-user case.

According to (Liu \& Li 2005a) and (Goldsmith 2005), if only one user with one receive antenna is considered $(K=N=1)$, the ergodic channel capacity can be expressed by: ${ }^{1}$

$$
C=\mathbb{E}_{H}\left[\log \left|\mathbf{I}_{1}+\frac{\mathbf{h Q h}^{H}}{\sigma^{2}}\right|\right]
$$

where $\mathbf{h}=\left[h_{1}, h_{2}, \ldots, h_{M}\right]$ is the channel matrix, $\mathbf{Q}$ is the transmit signal covariance matrix and $\sigma^{2}$ is the noise variance. Subject to a power constrain $P, \operatorname{Tr}(\mathbf{Q})=P, \mathbf{x}$ must be circularly symmetric complex Gaussian (Telatar 1999) and its correlation matrix, Q, must be diagonal $^{2}$ (Visotsky \& Madhow 2001), which is equivalent to independent transmit signals, for the ergodic channel capacity, $C$, to be maximal.

Taking into account that $\mathbf{Q}$ must be a diagonal matrix, $\mathbf{Q}=\operatorname{diag}\left(\left[P_{1}, P_{2}, \ldots, P_{M}\right]\right)$, where $P_{i}$ is the signal $x_{i}$ mean transmit power, and knowing that a single antenna user is considered, $N=1$, the ergodic capacity formula reduce to:

$$
C_{M}=\mathbb{E}_{H}\left[\log \left(1+\sum_{i=1}^{M} \frac{\left|h_{i}\right|^{2} P_{i}}{\sigma^{2}}\right)\right]
$$

From the previous expression it is easy to identify $\frac{\left|h_{i}\right|^{2} P_{i}}{\sigma^{2}}$ as the link $i \operatorname{SNR}\left(\gamma_{i}\right)$, then the capacity expression can be put into the following equivalent format: ${ }^{3}$

$$
C_{M}=\mathbb{E}_{\Gamma_{M}}[\log (1+\gamma)]=\int_{0}^{\infty} \log (1+\gamma) f_{\Gamma_{M}}(\gamma) d \gamma
$$

where $\Gamma_{M}$ is a random variable (RV) corresponding to the sum of $M$ exponential distributed RVs with mean $\lambda_{i}^{-1}$ each, which follow the pdf:

$$
f_{\Gamma_{M}}(\gamma)=\sum_{i=1}^{L} \sum_{n=1}^{K_{i}} \frac{a_{i n}}{(n-1) !} \gamma^{n-1} e^{-\lambda_{i} \gamma}
$$

1 We consider for now on that the capacity units are nats/s/Hz, when omitted.

2 The covariance matrix of the channel gains is diagonal, because the channel gains are independent. So their unitary singular value decomposition matrices are equal to the identity matrix.

3 According to (Goldsmith 2005) the $\left|h_{i}\right|^{2}$ random variable is exponential distributed and consequently $\gamma_{i}$. 
where $L$ is the number of different mean SNR's, $\lambda_{i}^{-1}$ is the mean SNR of link $i, K_{i}$ is the number of antennas with SNR $\lambda_{i}^{-1}, a_{i n}$ are constants related to the partial fraction expansion (Ghosh 2005) of the product of $I$. Erlang distribution characteristic functions (Gubner 2006) and $M=\sum_{i=1}^{L} K_{i}$. Finally, after the integral evaluation, (Dohler et al. 2006) or (Gradshteyn \& Ryzhik 1994), the ergodic channel capacity is given by:

$$
C_{M}=\sum_{i=1}^{L} \sum_{n=1}^{K_{i}} \sum_{k=0}^{n-1} \frac{a_{i n}}{\lambda_{i}^{n}} C_{i}\left(\lambda_{i}, k\right)
$$

where

$$
\begin{gathered}
C_{i}\left(\lambda_{i}, k\right)=\frac{\left(-\lambda_{i}\right)^{k}}{k !}\left[C_{0}\left(\lambda_{i}\right)+u(k-1) \sum_{p=1}^{k} \frac{(p-1) !}{\left(-\lambda_{i}\right)^{p}}\right] \\
C_{0}\left(\lambda_{i}\right)=e^{\lambda_{i}} E_{1}\left(\lambda_{i}\right)
\end{gathered}
$$

$C_{0}\left(\lambda_{i}\right)$ is equal to the capacity of the link associated with a single transmit antenna with SNR $\lambda_{i}^{-1}$ and is also equal to $C_{i}\left(\lambda_{i}, 0\right) . E_{1}(x)$ denotes the exponential integral function, given by $E_{1}(x)=\int_{x}^{\infty} e^{t} / t d t$ and $u(n)$ is the unit step function.

\subsection{Multi-User case}

In this section we briefly describe the broadcast channel sum-capacity and an affine approximation to this sum-capacity, in the high SNR regime. This approximation will be used in the following sections to analyze the DCAP for the multi-user case.

The broadcast channel capacity was over a large number of years an active area of research, which has culminated with an article by Weingarten et al., (Weingarten et al. 2006), where the authors have shown that the DPC rate region is the capacity region of the Gaussian MIMO BC. For the multi-user case the capacity expression is not as easy to analyze as the one for the single-user case, due to some additional constraints in the transmit covariance matrix and from the fact that there is no known closed form solution for this matrix that maximizes the channel capacity (Jindal et al. 2005).

From the MIMO BC-MAC duality, (Vishwanath et al. 2003), (Viswanath \& Tse 2003), (Wei Yu \& Cioffi 2004), the DPC sum rate with a total power constraint $P,\left(\sum_{k} \operatorname{tr}\left(\mathbf{Q}_{k}\right) \leq P\right)$, can be expressed, by:

$$
\mathbf{C}_{D P C}^{(M, K, N)}=\max _{\sum_{k} \operatorname{tr}\left(\mathbf{Q}_{k}\right) \leq P} \log \left|\mathbf{I}_{M}+\sum_{k=1}^{K} \mathbf{H}_{k}^{H} \mathbf{Q}_{k} \mathbf{H}_{k}\right|=\max _{\operatorname{tr}(\mathbf{Q}) \leq P} \log \left|\mathbf{I}_{M}+\mathbf{H}^{H} \mathbf{Q H}\right|
$$

where $\mathbf{Q}_{k}\left(\in \mathbb{C}^{N \times N}\right)$ is the transmit covariance matrix in the dual MAC channel and $\mathbf{Q}=\operatorname{diag}\left(\mathbf{Q}_{1}, \ldots, \mathbf{Q}_{K}\right)$ is a block-diagonal matrix. In the high SNR regime and for $K N \leq M$, equal power allocation, $\mathbf{Q}_{k}=\frac{P}{K N} \mathbf{I}_{N}$, is asymptotically optimal (Juyul Lee \& Jindal 2007). As a byproduct of that, the following affine approximation to the sum-capacity can be made (Juyul Lee \& Jindal 2007), (Shamai \& Verdu 2001): 


$$
\mathbf{C}_{D P C}^{(M, K, N)} \approx K N \log P-K N \log K N+\log \left|\mathbf{H} \mathbf{H}^{H}\right|
$$

From this affine approximation it is easy to see that the sum-capacity scales linearly with the number of aggregate receive antennas and that this scaling factor is not dependent on the individual values of $K$ or $N$, in the high SNR regime. However, for the case of more aggregate receive than transmit antennas, $K N>M$, equal power allocation ceases to be asymptotic optimal, since even for the standard degraded BC $(M=1)$ the throughput is maximized by transmitting only to the best user (Caire \& Shamai 2003).

\section{Single-User DCAP}

\subsection{Exact Expression}

In this sub-section we obtain an exact expression for the DCAP considering the the singleuser scenario. During our analysis of the DCAP behavior it will be observed that the DCAP tends to have a small decay relatively to the previous DCAP value, when the new added antenna has the same mean SNR of a previously connected antenna. In that context we also derive an expression for the DCAP that contains explicitly the value of the previous DCAP to, latter on, do an analysis of the sensitivity of the DCAP values versus the mean SNR of the new connected antenna, as more and more antennas are connected to the system.

From the definition of the $\Gamma_{M} \mathrm{RV}$ it is possible to prove that:

$$
f_{\Gamma_{M}}(\gamma)=\lambda_{M} e^{-\lambda_{M} \gamma} \int_{0}^{\gamma} f_{\Gamma_{M-1}}(x) e^{\lambda_{M} x} d x
$$

with which a recursive algorithm for the calculation of the $a_{i n}$ coefficients, which are central to the calculation of the capacity values, equation (6), can be obtained, and the following formula can be derived:

$$
-\frac{d f_{\Gamma_{M}}(\gamma)}{d \gamma}=\lambda_{M}\left[f_{\Gamma_{M}}(\gamma)-f_{\Gamma_{M-1}}(\gamma)\right]
$$

From equation (10), after multiplying by $\log (1+\gamma)$ and integrating from zero to plus infinity the $\gamma$ variable, at both sides of the equation, the DCAP can be expressed by:

$$
\begin{aligned}
\Delta C_{M-1}^{M} & =C_{M}-C_{M-1}=-\lambda_{M}^{-1} \int_{0}^{\infty} \frac{d f_{\Gamma_{M}}(\gamma)}{d \gamma} \log (1+\gamma) d \gamma \\
& =\lambda_{M}^{-1} \int_{0}^{\infty} \frac{f_{\Gamma_{M}}(\gamma)}{1+\gamma} d \gamma=\lambda_{M}^{-1} \sum_{i=1}^{L} \sum_{n=1}^{K_{i}} \frac{a_{i n}}{\lambda_{i}^{n-1}} C_{i}\left(\lambda_{i}, n-1\right)
\end{aligned}
$$

With some more mathematical manipulations, the differential capacity can also be equivalently expressed by:

$$
\begin{aligned}
\Delta C_{M-1}^{M} & =\frac{\lambda_{M-1}}{\lambda_{M}} \Delta C_{M-2}^{M-1} \\
& +\frac{1}{\lambda_{M}^{2}}\left[C_{0}\left(\lambda_{M}\right) f_{\Gamma_{M}}(0)-\int_{0}^{\infty} \frac{f_{\Gamma_{M}}(\gamma)}{(1+\gamma)^{2}} d \gamma\right]
\end{aligned}
$$


where $f_{\Gamma_{M}}(0)$ is equal to 0 for $M>1$ and equal to $\lambda_{1}$ for $M=1$. This expression will be used in the following sections to analyze the DCAP sensitivity with the number of transmit antennas and the mean SNR's of the new connected antennas.

\subsection{DCAP Bounds}

Even if the previous expressions obtained for the DCAP, namely equation (11) and (12), are exact they are not easy to analyze, mostly because of the presence of the $a_{i n}$ coefficients. Therefore the derivation of some simple upper/lower bounds to the DCAP is an important step to analyze its behavior. For doing that, we will rely mostly on the second integral definition of the DCAP, from equation (11). From that expression one can easily see that if some bounds for equation $g(\gamma)=1 /(1+\gamma)$ are available, they can be easily used to bound also the DCAP. One simple bound for equation $g(\gamma)$ is $g(\gamma) \leq 1$, for all $\gamma$ in $[0, \infty[$. As a consequence the DCAP can be upper bounded by:

$$
\Delta C_{M-1}^{M} \leq \frac{1}{\lambda_{M}} \int_{0}^{\infty} f_{\Gamma_{M}}(\gamma) d \gamma=\lambda_{M}^{-1}
$$

and the channel capacity can be upper bounded by:

$$
C_{M} \leq \sum_{k=1}^{M} \lambda_{k}^{-1}=\sum_{i=1}^{L} K_{i} \lambda_{i}^{-1}
$$

Another possible bound for equation $g(\gamma)$ can be obtained from $1+x \leq e^{x}$, for all $x$ in $[0, \infty[$, by the Taylor series expansion of the exponential function around zero. Thus $g(\gamma) \leq e^{-\gamma}$, for all $\gamma$ in $\left[0, \infty\left[\right.\right.$, with a maximum difference of $\left(M_{d} \approx 0.204\right)^{4}$, so:

and as a byproduct:

$$
e^{-\gamma} \leq g(\gamma) \leq e^{-\gamma}+M_{d}
$$

$$
\frac{\varphi_{\Gamma_{M}}(-1)}{\lambda_{M}} \leq \Delta C_{M-1}^{M} \leq \frac{\varphi_{\Gamma_{M}}(-1)}{\lambda_{M}}+\frac{M_{d}}{\lambda_{M}}
$$

where $\varphi_{\Gamma_{M}}(s)$ is the $\Gamma_{M} \mathrm{RV}$ moment generating function.

From bound (13) and (16) follows that in the low SNR regime the DCAP can be approximated by $\lambda_{M}^{-1}$ and the channel capacity by:

$$
C_{M} \approx \sum_{i=1}^{L} K_{i} \lambda_{i}^{-1}
$$

The previous bounds although simple can provide some interesting information on the behavior of the DCAP, as will be seen in section 6.1. However they do not give any idea on the SNR vector that attain the maximum of the DCAP. This vector can give information on how the distributed antennas should be geographically positioned to attain most of the gains provided by the connection of additional antennas to the system. In the following paragraphs we answer this question, and prove that the SNR vector that attains the highest

\footnotetext{
${ }^{4}$ Obtained numerically, and knowing that this maximum is global.
} 
DCAP value is the SNR vector where all elements are equal. In other words the highest DCAP is obtained when all transmit antennas are co-located, have the same link SNR

From equation (11) one can see that $d \Delta C_{M-1}^{M} / d \lambda_{i} \geq 0$ for $M>1$ and $i \neq M$. Therefore if we assume that the new connected antenna is always the one with the highest mean SNR, the DCAP will be upper bounded by the one where all mean SNRs are equal to the mean SNR of the new connected antenna:

$$
\begin{gathered}
\Delta C_{M-1}^{M}\left(\lambda_{1}, \ldots, \lambda_{M}\right) \leq \Delta C_{M-1}^{M}\left(\lambda_{M}, \ldots, \lambda_{M}\right) \\
\lambda_{M} \geq \lambda_{n}, \forall n \in[1,2, \ldots, M-1]
\end{gathered}
$$

When the mean SNR tends to infinity the maximum of the DCAP is obtained and is equal to:

$$
\lim _{\lambda_{M} \rightarrow 0} \Delta C_{M-1}^{M}\left(\lambda_{M}, \ldots, \lambda_{M}\right)=\frac{1}{M-1}
$$

Thus the DCAP in general is upper bounded by the co-located transmit antennas case and its maximal value is only dependent on the number of transmit antennas and not on the actual values of the mean SNR of the links. However this limit is not attainable in practice. But, how far is it from a real scenario? It can be shown that for a moderate SNR of $17 \mathrm{~dB}$, for all links, the difference is lower than $0.1 \mathrm{bps} / \mathrm{Hz}$ when we pass from one connected antenna to two and even lower for the other connected antennas.

Since the previous bound is a limit bound it can be not very tight. However a tighter bound can be developed considering only a slight higher degree of information:

$$
\Delta C_{M-1}^{M} \leq \frac{1}{\lambda_{M}+M-1} \leq \frac{1}{M-1}
$$

From the previous expression one can also upper bound the capacity increase by the connection of $J$ new antennas, considering that the user is actually only connected to one antenna and that antenna has the greatest SNR of all of them, by:

$$
\Delta C_{1}^{J+1} \leq \sum_{n=2}^{J+1} \frac{1}{n-1}=\sum_{n=1}^{J} \frac{1}{n} \approx \gamma+\log (J+\beta)
$$

where $\gamma$ is the Euler constant $(0.577215667)$ and $\beta=e^{1-\gamma}-1$. Consequently as the number of connected antennas increase the DCAP decreases. However, if the number of antennas grows to infinity so does the capacity, but of course, in practice, one can only have a limited number of antennas. Thus a tradeoff between the cost and capacity gains must be made when we choose the number of antennas to be deployed in a real system.

\section{Multi-User DCAP}

In the previous section we have analyzed the DCAP for the single-user case and have provided an exact closed form expression for it, that is valid for all mean SNR's. We have started with the single user case due to its simplicity and also because the analysis of that simpler case can give some insight for the analysis of the more difficult case of a multi-user 
system. However for the more general case of a multi-user system the analysis of the DCAP over all mean SNR's is intractable. Thus, for this case, we consider only the DCAP in the high SNR regime. In this section we first define the DCAP and after derive a closed form expression for it, for the co-located antenna case and for $M \geq K N$. For $K N>M$ the DCAP is analyzed numerically.

According to equation (8), for $M \geq K N$, the DCAP can be expressed by:

$$
\begin{aligned}
\Delta C_{M, K, N}^{M+1, K, N} & =\mathbb{E}\left[\mathbf{C}_{D P C}^{(M+1, K, N)}-\mathbf{C}_{D P C}^{(M, K, N)}\right] \\
& =\mathbb{E}\left[\log \left|\overline{\mathbf{H}}_{M+1} \overline{\mathbf{H}}_{M+1}^{H}\right|\right]-\mathbb{E}\left[\log \left|\overline{\mathbf{H}}_{M} \overline{\mathbf{H}}_{M}^{H}\right|\right]
\end{aligned}
$$

where $\overline{\mathbf{H}}_{i},(i=M, M+1)$ is the channel matrix for $i$ transmit antennas. Thus for the multiuser scenario the DCAP is only dependent on the matrix $\mathbf{H}$ distribution. On the other hand, for $K N>M$, the DCAP can be expressed by:

$$
\begin{aligned}
\Delta C_{M, K, N}^{M+1, K}, N & =\mathbb{E}\left[\mathbf{C}_{D P C}^{(M+1, K, N)}-\mathbf{C}_{D P C}^{(M, K, N)}\right] \\
& =\mathbb{E}\left[\mathbf{C}_{D P C}^{(M+1, M+1,1)}+\Delta C_{M+1, M+1,1}^{M+1, K, N}-\left(\mathbf{C}_{D P C}^{(M, M, 1)}+\Delta C_{M, M, 1}^{M, K, N}\right)\right] \\
& =\Delta C_{M, M, 1}^{M+1, M+1,1}+\Delta C_{M+1, M+1,1}^{M+1, K, N}-\Delta C_{M, M, 1}^{M, K, N} \\
& =\log (P)+\Delta
\end{aligned}
$$

From which we can see that the connection of the system users to a new transmit antenna is equal to $\log (P)$, the multiplexing gain, plus a $\Delta$ factor, that is equal to $\Delta C_{M+1, M+1,1}^{M+1, K, N}-\Delta C_{M, M, 1}^{M, K, N}$ plus the power offset gain provided when we pass from $M$ to $M+1$ transmit antennas and users ${ }^{5}$, which can be closely approximated by $-\log (e)$, for any number of transmit antennas. Hence, the significance of the $\Delta$ factor versus the multiplexing gain must be analyzed to have a clear picture of the full obtained gain, please refer to section 5.2 and 6.2 .

\subsection{DCAP for $M>K N$}

In this sub-section the DCAP for the optimal scheme, DPC, and for two sub-optimal schemes, namely ZF and BD is analyzed. A closed form expression and an approximation for it are also derived. Unfortunately, the general case of a distributed antenna system is difficult to analyze mathematically, thus for this case we will relie on numerical analysis, which are provided in section 6.2. In the analysis, contained in this sub-section, we consider that all transmit antennas are co-located.

For DPC, as shown in appendix (sub-section 9.1), the DCAP when we pass from $M$ to $M+1$ transmit antennas is given by:

$$
\begin{aligned}
{ }_{5} \Delta C_{M, M, 1}^{M+1, M+1,1} & \approx \log (P)+\log (M+\beta)+M \log M-(M+1) \log (M+1) \\
& \approx \log (P)-\log (e)
\end{aligned}
$$




$$
\begin{aligned}
\Delta C_{M, K, N}^{M+1, K, N} & =\sum_{n=M-K N+1}^{M} \frac{1}{n}=\sum_{n=M-K N+1}^{M} \Delta C_{n, 1,1}^{n+1,1,1} \\
& \approx \log \left(1+\frac{K N}{M-K N+\beta}\right)
\end{aligned}
$$

where $\beta=e^{1-\gamma}-1$ and $\gamma=0.577215665$ is the Euler constant. For the linear precoding schemes a similar expression can be obtained, by noting that for BD the DCAP is equal to $K$ times the DPC one, for a $(M-(K-1) N) \times N$ channel, from the BC equivalent point to point MIMO interpretation, in the high SNR regime (Juyul Lee \& Jindal 2007). For that reason, the $\mathrm{DCAP}$ for $\mathrm{ZF} / \mathrm{BD}$ can be show to be equal to:

$$
\overline{\Delta C}_{M, K, N}^{M+1, K} \approx K \log \left(1+\frac{N}{M-K N+\beta}\right)
$$

From the previous expression one can see that asymptotically in the number of transmit antennas, $M$, the DCAP behaves like $\log _{2}(e) K N /(M-K N+\beta)$, for both DPC and ZF/BD, implying that the difference between the gains of the optimal scheme and the sub-optimal schemes is very small, when a high number of transmit antennas is considered. Nevertheless for a finite number of transmit antennas the DCAP gains provided by DPC are lower and the difference increases with $K$, as can be seen by equations (24) and (25). But how it scales with $K$, the number of users? It can be seen that for $M-K N$ a constant the DCAP increases logarithmically with $K$ for DPC and linearly with $K$ for ZF/BD. Thus one can conclude that even if the optimal scheme has a higher complexity, it will need a much lower number of transmit antennas than the sub-optimal schemes, which require a lower complexity, for the same target capacity. Thus the number of antennas to be jointly processed must be carefully chosen, taking into account the tradeoff between complexity, network costs and obtained benefits.

\subsection{DCAP for $\mathrm{KN}>\mathrm{M}$}

In this sub-section the DCAP is analyzed for the case of more aggregate receive than transmit antennas. For this case, since equal power allocation stop to be asymptotically optimal and no closed form solution exists for the power allocation problem (Jindal et al. 2005), a mathematical study to attain a closed form expression for the DCAP is not possible. Nevertheless an approximation for this DCAP can be obtained. But how? It is know that for the case of only one transmit antenna, $M=1$, it is optimal to transmit only to the best user (Caire \& Shamai 2003) and as proven in the appendix (sub-section 9.2), for this case the maximum increase in capacity, when one additional user is connected to the system, is attained when all users are co-located i.e. when they have equal mean link SNR's. For $M=1$ and $N=1$ and for the high SNR regime this maximum is equal to, see appendix (sub-section 9.2):

$$
\Delta C_{1, K, 1}^{1, K+1,1}=\sum_{n=0}^{K}\left(\begin{array}{c}
K \\
n
\end{array}\right)(-1)^{K+1} \log (K+1)
$$

At this point we have only obtained an expression for the capacity increase by the connection of one additional system user, for the case of only one transmit antenna. But to 
obtain an approximation for the DCAP, for $K N>M$, we need also to know that for $M>1$, see equation (23). For $M>1$ the increase in capacity obtained by the connection of a new user to the system, can be approximated taking into account the DCAP expression for the $M>K N$ case, equation (24) line 1, and making an extrapolation of equation (26). From those assumptions the capacity increase by the connection of one additional user to the system can be approximated by:

$$
\Delta C_{M, K, 1}^{M, K+1,1} \approx \sum_{n=K-M+1}^{K} \Delta C_{1, n, 1}^{1, n+1,1}
$$

and as can be confirmed in sub-section 6.2 (Fig. 3(a)), this approximation is close to the values obtained by numerical simulations and is better for a high number of users.

\section{Numerical Results}

\subsection{Single-User DCAP analysis}

In this sub-section, the DCAP, for a grid antenna placement, as show in Fig. 3 (a), is analyzed. First the exact DCAP values are compared to the obtained bounds, to access their tightness. Next we analyze the sensitivity of the DCAP to the variation of the mean SNR and finally we perform a DCAP analysis of a representative area covered by the antennas.

In all numerical analysis presented in this chapter we consider that the mean SNR is only dependent on the signal path loss (Simplified Path Loss Model, (Goldsmith 2005)), that ${ }^{6}$ $P_{t} K d_{0}^{\gamma} / \sigma_{n}^{2}=1$ and that $\gamma=3$. It is also considered that the new connected antenna is always the one with highest mean SNR from the group of unconnected antennas and that $\Delta d_{x}=\Delta d_{y}=\Delta d$. By circular ring we mean the group of antennas with the same SNR.

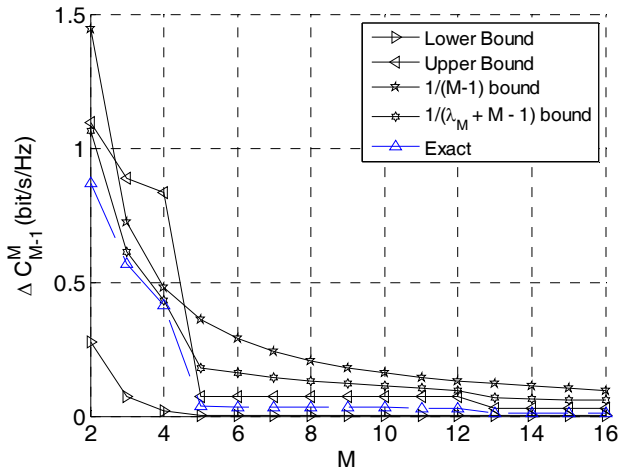

(a) Central Point $\Delta d=1$.

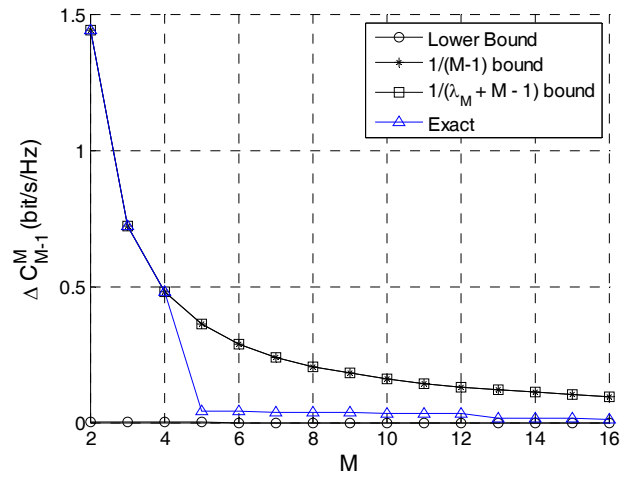

(b) Central Point $\Delta d=0.1$.

Fig. 1. Differential capacity by the connection to one more antenna, exact, upper and lower bounds.

${ }^{6} K$ is a unit less constant which depends on the antenna characteristics and on the average channel attenuation, $d_{\mathrm{Q}}$ is a reference distance for the antenna far-field, $\gamma$ is the path loss exponent and $P_{t}$ is the transmitted power at a distance $d_{\mathbb{a}}$. 
In Fig. 1 we plot the exact DCAP values and respective bounds for the central point of the grid antenna placement and do that for two different inter-antenna distances, namely $\Delta d=1$ and $\Delta d=0.1$. From this figure one can see that if the new connected antenna is in the same circular ring as a previously connected antenna then its DCAP value, $\Delta C_{M}^{M-1}$, will be approximately the same as the previous one, $\Delta C_{M-2}^{M-1}$. On the other hand if the new connected antenna is far away from the circular ring of a previously connected antenna then the DCAP value decreases a lot. This approximation is better when a high number of transmit antennas is considered. Thus one can conclude intuitively that if all antennas are in the same circular ring, have the same mean SNR, their DCAP values will be maximum. Indeed this is true as shown in section 4.2. This behavior can be explained by the fact that $\Delta C_{M-1}^{M}$ is dependent on $\Delta C_{M-2}^{M-1}$ by a factor of $\lambda_{M-1} / \lambda_{M}$, an approximation that due to its importance will be analyzed in the following paragraphs. It can also be explained by the fact that in equation (16), $\varphi_{\Gamma_{M}}(-1)$ tends to zero as we connect to more antennas and as a consequence the bound becomes independent off all SNR's except the new one, which for a circular ring is constant. Thus, since the central point in the grid antenna placement is the one with highest symmetry we can say that if a target increase in capacity is pre-established then the user will be connected to 4 or 12 or 16 or 24 or 32 or . . antennas, depending on the target increase in capacity defined.

Concerning the bounds tightness we can see from that figure that for low SNR's the upper bound provided by equation (16) is the better one, but for high SNR's the bound from equation (20) is more accurate. Although, the user will only connect to a small number of antennas in a real system, due to the diminishing returns expected as the number of antennas increase. Thus, taking into account this fact, the most important bounds will be the ones that are tighter for a low number of transmit antennas, and in this case the winner is the bound from equation (20).

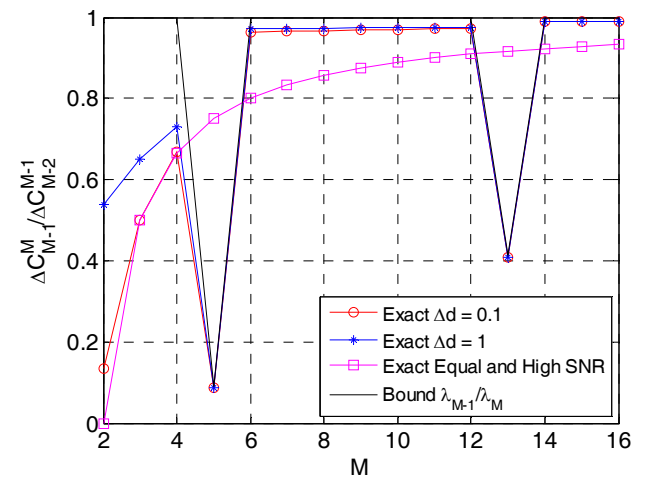

(a) Central Point.

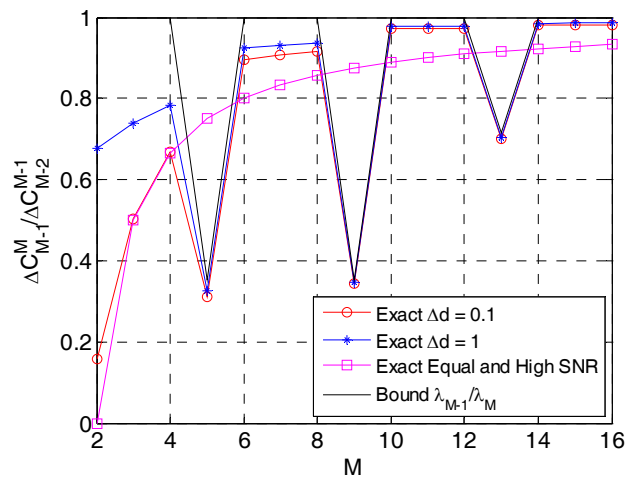

(b) $\left(0.5 \Delta d_{x}, 0.5 \Delta d_{y}\right)$ Point.

Fig. 2. Differential capacity sensitivity with respect to $M$ and for different inter-antenna distances.

From Fig. 1 (a) and Fig. 1 (b) one can also see that the exact values of the DCAP, the dashed and solid blue lines, converge to the same value, for a high number of transmit antennas in the case of SNR vectors that are multiple among themselves. This fact can be easily proven 
together with the fact that as the SNR's get higher this convergence occurs at a smaller $M$ value. In the case of high SNR's the DCAP cannot be higher than a given value, having as critical value $1 /(M-1)$, in the case of equal SNR's. As a consequence, if all transmit antennas are put closer to the user terminal, by a given factor, the only antennas for which the DCAP increases are the ones near the terminal.

As previously verified the DCAP in a circular ring tends to be constant. But how close to the previous value and when this approximation can be made? In the following paragraphs we propose to analyze this fact using a bound for the ratio $\Delta C_{M-1}^{M} / \Delta C_{M-2}^{M-1}$. If the ratio is close to one then the $\Delta C_{M-1}^{M}$ value will be close to $\Delta C_{M-2}^{M-1}$. In Fig. 2 (a) and Fig. 2 (b) we plot this ratio for two different points in the grid antenna placement, namely for the central point and for $\left(0.5 \Delta d_{x}, 0.5 \Delta d_{y}\right)$. In each figure the exact ratio value for two inter-antenna distances $(\Delta d=1$ and $\Delta d=0.1)$, the bound from equation (12) and the exact value for equal and high SNR's are plotted. From those results one can view that as the number of connected transmit antennas increase the ratio sensitivity to the SNR vector variation decreases and that its exact value becomes closer to the bound. One can also see that when the new connected antenna is not from the same ring as the previously connected antenna, the bound is indeed very close to the actual value of the respective ratio and the ratio value as a step decrease that is lower as more connected antennas are considered. As a consequence the DCAP tends to be constant in a circular ring, as previously observed, and the approximation is better when a high number of transmit antennas is considered.

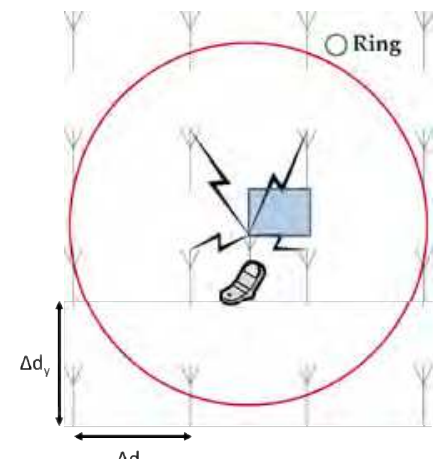

(a) Geographical antenna placement.

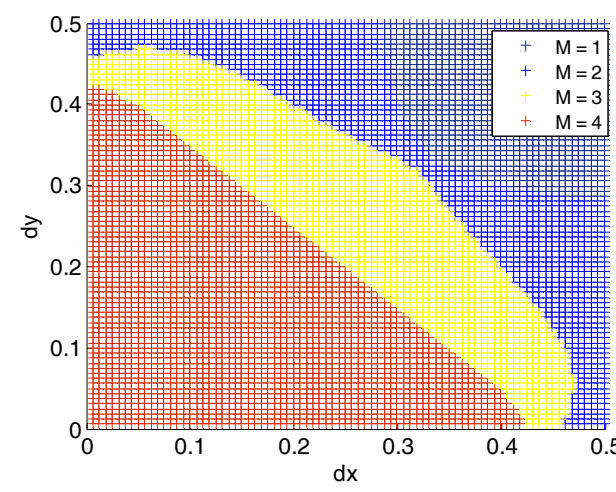

(b) Number of antennas where DCAP is higher than $0.1 \mathrm{bps} / \mathrm{Hz}$.

Fig. 3. Single-User differential capacity analysis, for a regular antenna placement.

Thus far, we have only analyzed the DCAP values for a single point, but how it will behave if we consider a given area. The analysis of the DCAP values for a given area cannot be easily visualized in a two dimensional plot, thus we have relied on a different measure to analyze its behavior. The metric considered was the maximum number of transmit antennas that achieve a target DCAP value or more. In this study we have considered a target DCAP value of $0.1 \mathrm{bps} / \mathrm{Hz}$. The geographical area considered was the one shown in figure Fig. 3 (a) in light blue, and we have also considered $\Delta d_{x}=\Delta d_{y}=1$. The results of this study are presented on figure Fig. 3 (b). To explain the information contained into this figure it is 
easier to give an example. Thus, let's assume, for example, the point $(0.4,0.4)$. For this point $M=4$, thus when we connect the second, third and fourth antennas the DCAP value is higher than the target, but departing from that number of transmit antennas, 4 , the DCAP will be lower than the target.

For the considered scenario and for a target DCAP of $0.1 \mathrm{bps} / \mathrm{Hz}$ only the first four antennas will be connected to the user, or equivalent the antennas presented in the first ring. This figure also shows a circular pattern in the number of necessary antennas. This is related to the fact that at a given distance the link mean SNR is only dependent on the distance from the user to the considered transmit antenna.

\subsection{Multi-User DCAP analysis}

In this sub-section the DCAP of DPC is compared to the one of ZF/BD, for the co-located antennas scenario. For the DAS, as stated before, we rely on numerical simulations to access the capacity gains provided by the connection of additional transmit antennas. For this numerical analysis we consider a scenario with 4 transmit antennas and 2 users, each with only one receive antenna. Finally for $K N \geq M$ we analyze the DCAP for the equal mean SNR's case. But to do that we first analyze the capacity gain provided by the connection of an additional user to the system and after that we investigate the gain provided by $\Delta$ in comparison to $\log (P)$, see equation (23).

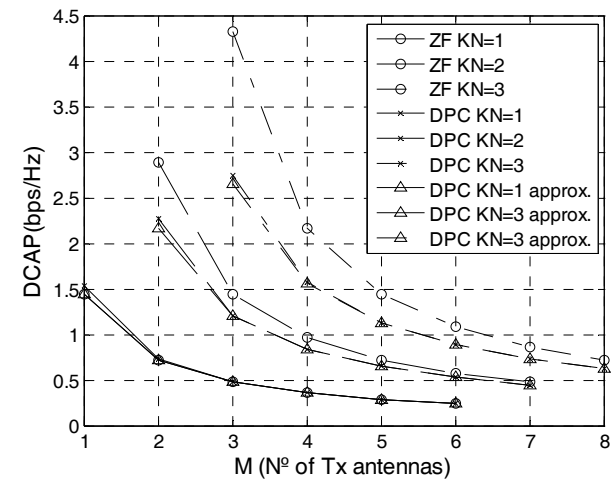

(a) DPC versus ZF.

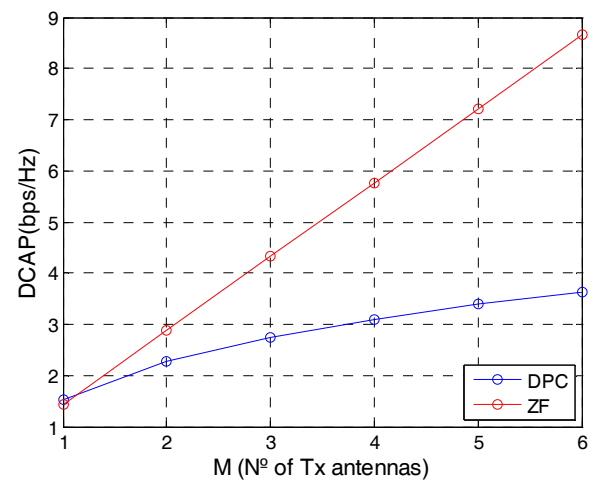

(b) DCAP for $M=K N$.

Fig. 4. Differential capacity for different number of user's and receive antennas.

As can be seen in Fig. 4 (a), were we present a plot of the DPC and ZF DCAP versus the number of connected transmit antennas, for the co-located scenario, the logarithm approximation although simple is very tight. Concerning the benefits of the connection of new transmit antennas, ZF has higher gains than DPC and the difference increase with $K N$. To see how this difference scales with $K$ we plot in Fig. 4 (b) the DCAP values versus $M$ for $M=K N$. From this figure one can view that the DCAP scales logarithmically with the number of users for DPC and linearly for ZF, as already seen from equations (24) and (25). Thus even if the implementation complexity of DPC is much higher than the one for $\mathrm{ZF}$, the sub-optimal scheme, ZF, will need a higher number of transmit antennas than the optimal 
scheme for a target system capacity. Thus a tradeoff must be made between the cost of additional processing power and the cost of additional physical resources, namely transmit antennas and respective connection to the central unit. As a result of this analysis, as a scheme approaches the optimal one, the gains obtained by the connection of additional antennas at the transmitter side decrease. But, remember that the optimal scheme will have always a higher sum-capacity. As seen before, from equations (24) and (25), the DCAP for the two precoding schemes converge to the same value for a high number of transmit antennas and the convergence point increases with $K N$.

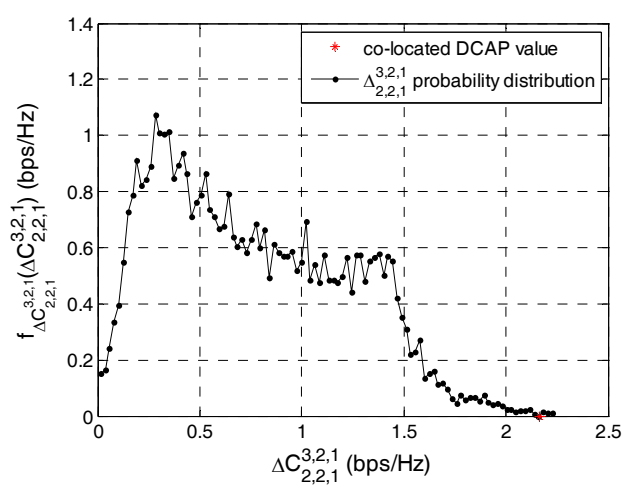

(a) DCAP distribution for $M=2$.

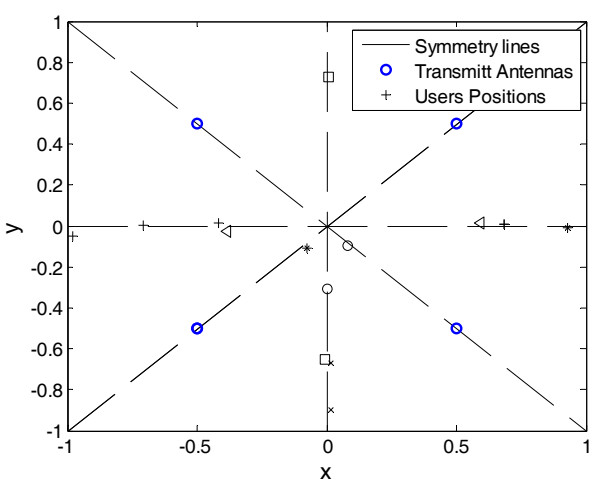

(b) User's position, where distributed DCAP is higher than co-located DCAP.

Fig. 5. Differential capacity distribution for a uniform user distribution and user's positions where the distributed DCAP is higher than the co-located DCAP. Each pair of equal black markers represent the positions of each user.

For the DAS we will only analyze the DCAP for DPC and that analysis will rely on numerical simulations. For this simulation a scenario with 4 transmit antennas and two users, each with only one receive antenna, is considered. To model the channel we have considered the Simplified Path Loss Model again, (Goldsmith 2005), with a path loss exponent equal to 3, and have considered Rayleigh multipath fading. The transmit antennas were positioned as shown in Fig. 5 (b) and the user positions were randomly generated with an uniform distribution in $[-1,1] \times[-1,1]$. Ten thousand user's positions were generated and the DCAP was averaged over 10000 trials. The new connected antennas were always chosen to be the one that will imply the highest DCAP from the group of unconnected antennas. As a result of this simulation, we have Fig. 5, where the co-located DCAP, equation (24), value is represented by a red star. From that figure we see that most of the user's positions have a smaller DCAP than the co-located case. However for a small elite of users, 7 in this specific case, the DCAP is higher than the one obtained by the co-located scenario. Thus one can conclude that even if the DCAP for a DAS can be higher than the one for the co-located case the difference will not be high and the number of user positions in that condition will be low. To have a better understanding of the positions that attain the maximum DCAP gains for a DAS, in Fig. 5 (b), we show the 7 positions that have a higher DCAP than the co-located case. From that figure it is possible to infer that these positions 
are all very close to the system symmetry lines defined by the four transmit antennas and in that way, as in the co-located case, the new connected antenna will have the same distribution as a previous one. Thus system symmetry plays an important role in obtaining most of the DCAP gains, either for the single-user either for the multi-user cases.

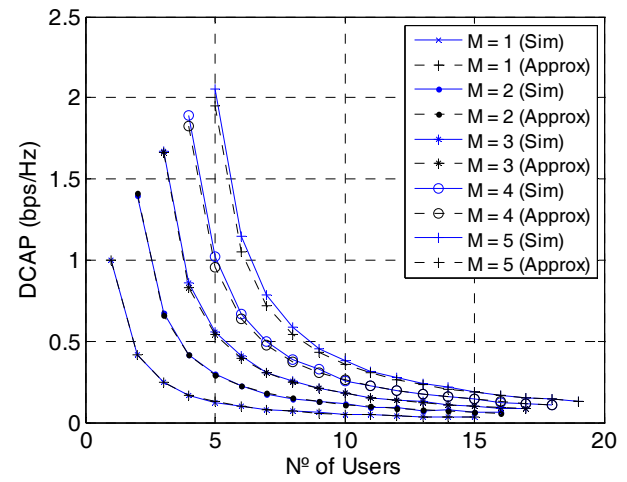

(a) Capacity increase by the connection of a new user $(\mathrm{N}=1)$.

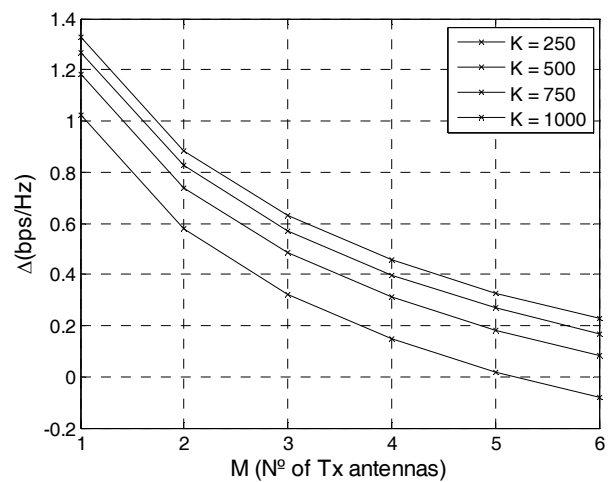

(b) $\Delta$ factor value $(\mathrm{N}=1)$.

Fig. 6. Capacity increase by the connection of one more transmit antenna to the system, for $K N>M$.

When more aggregate receive than transmit antennas are considered it is important to analyze the $\Delta$ factor in equation (23), to see if it has some significance in relation to $\log _{2}(P)$. Thus, we must evaluate the capacity increase obtained by the connection of an additional user to the system. To do that, we have relied on numerical simulations, considering that $N=1$ and that each column of $\mathbf{H}$ is $\tilde{\mathcal{N}}_{K N}(0, \mathbf{I})$ distributed. Since for this case no closed form expression exists for the power allocation we have relied on the algorithm presented in (Jindal et al. 2005) to optimally allocate power among users. As a result Fig. 6 (a) the ergodic capacity increase, when a new user is connected to the system, was obtained... In Fig. 6 (a)it is also shown the equation (27) approximation, which is very close to the obtained numerical results. Taking into account that approximation in our analysis, in Fig. 6 (b), we plot the $\Delta$ factor values for different number of users. From those values one can see that even for 1000 users this factor is small, not higher than $1.4 \mathrm{bps} / \mathrm{Hz}$. Thus it has no significance in relation to $\log (P)$, since we are considering the high SNR regime. From that figure we can also confirm that $\Delta$ increases fewer as $K$ increases. Consequently, to obtain a $\Delta$ value with the same order of magnitude of $\log (P)$ a very high number of users should be jointly processed, which will result in a very high processing complexity at the CU, which must be avoided.

\section{Conclusion}

In this chapter we have analyzed the benefits of the connection of additional transmit antennas to the system users of a BC. The single-user case has been analyzed in more detail, for all SNR's and the multi-user case has been analyzed only in the high SNR regime. For 
the single user case we have obtained a closed-form expression for DCAP that is valid for all SNR's and have analyzed its variation/sensitivity with respect to $M$. From that analysis we have seen that symmetry is the most important system property to obtain most of the gains provided by the connection of additional transmit antennas. It was also shown that the maximum increase in capacity is obtained when all links have the same mean SNR's and not when they are different. Thus showing that the DCAP maximum is obtained when all antennas are co-located and not distributed.

For the multi-user case a closed form expression for the co-located transmit antennas DCAP, for $M \geq K N$, has been obtained. For the general case of a DAS the DCAP was analyzed numerically, considering a scenario with 4 transmit antennas and 2 users, each with only one receive antenna. For this case even if the DCAP can be superior to the co-located case it is not much higher and most of the user's positions will have a lower DCAP than the colocated case. For the positions that attain a higher DCAP than the co-located case we have shown that this positions are very close to the system symmetry lines, defined by the transmit antennas, and in that way symmetry plays again a important role in the multi-user case. For the case of more aggregate receive than transmit antennas we have verified that the DCAP will be power dependent and will be given by the multiplexing gain plus a $\Delta$ factor. However this $\Delta$ factor is much smaller than the multiplexing gain even for one thousand users. Thus for this case the most significant gain is the multiplexing gain, $\log (P)$.

\section{References}

Caire, G. \& Shamai, S., 2003. On the achievable throughput of a multiantenna Gaussian broadcast channel. Information Theory, IEEE Transactions on, 49(7), 1691-1706.

Castanheira, D. \& Gameiro, A., 2008. Distributed MISO system capacity over Rayleigh flat fading channels'. In Personal, Indoor and Mobile Radio Communications, 2008. PIMRC 2008. IEEE 19th International Symposium on. pp. 1-5.

Castanheira, D. \& Gameiro, A., 2009. High SNR Broadcast Channel Differential Capacity. In ICT-MobileSummit 2009. Santander, Spain, pp. 1-5.

Cover, T., 1972. Broadcast channels. Information Theory, IEEE Transactions on, 18(1), 2-14.

Dohler, M., Gkelias, A. \& Aghvami, A., 2006. Capacity of distributed PHY-layer sensor networks. Vehicular Technology, IEEE Transactions on, 55(2), 622-639.

FUTON, 2008. FUTON - Fibre-Optic Networks for Distributed Extendible Heterogeneous Radio Architectures and Service Provisioning. Available at: http://www.ictfuton.eu/.

Ghosh, S., 2005. Network Theory, PHI Learning Pvt. Ltd.

Goldsmith, A., 2005. Wireless communications, Cambridge University Press.

Gradshteyn, I.S. \& Ryzhik, I.M., 1994. Table of Integrals, Series, and Products 5th ed., Academic Press.

Gubner, J.A., 2006. Probability and Random Processes for Electrical and Computer Engineers 1st ed., Cambridge University Press.

I. R. R. M. M1645, 2003. M.1645: Framework and overall objectives of the future development of IMT-2000 and systems beyond IMT-2000. Available at: http:/ / www.itu.int/rec/R-REC-M.1645/e.

Jindal, N., 2005. High SNR analysis of MIMO broadcast channels. In Information Theory, 2005. ISIT 2005. Proceedings. International Symposium on. pp. 2310-2314. 
Jindal, N. et al., 2005. Sum power iterative water-filling for multi-antenna Gaussian broadcast channels. Information Theory, IEEE Transactions on, 51(4), 1570-1580.

Juyul Lee \& Jindal, N., 2007. High SNR Analysis for MIMO Broadcast Channels: Dirty Paper Coding Versus Linear Precoding. Information Theory, IEEE Transactions on, 53(12), 4787-4792

Liu, H. \& Li, G., 2005a. OFDM-Based Broadband Wireless Networks: Design and Optimization, Wiley-Interscience.

Liu, H. \& Li, G., 2005b. OFDM-Based Broadband Wireless Networks: Design and Optimization, Wiley-Interscience.

Seijoon Shim et al., 2008. Block diagonalization for multi-user MIMO with other-cell interference. Wireless Communications, IEEE Transactions on, 7(7), 2671-2681.

Shamai, S. \& Verdu, S., 2001. The impact of frequency-flat fading on the spectral efficiency of CDMA. Information Theory, IEEE Transactions on, 47(4), 1302-1327.

Shin, H. \& Lee, J.H., 2003. Capacity of multiple-antenna fading channels: spatial fading correlation, double scattering, and keyhole. Information Theory, IEEE Transactions on, 49(10), 2636-2647.

Telatar, I.E., 1999. Capacity of multi-antenna Gaussian channels. Available at: http://eprints.kfupm.edu.sa/29242/.

Vishwanath, S., Jindal, N. \& Goldsmith, A., 2003. Duality, achievable rates, and sum-rate capacity of Gaussian MIMO broadcast channels. Information Theory, IEEE Transactions on, 49(10), 2658-2668.

Visotsky, E. \& Madhow, U., 2001. Space-time transmit precoding with imperfect feedback. Information Theory, IEEE Transactions on, 47(6), 2632-2639.

Viswanath, P. \& Tse, D., 2003. Sum capacity of the vector Gaussian broadcast channel and uplink-downlink duality. Information Theory, IEEE Transactions on, 49(8), 1912-1921.

Wei Yu \& Cioffi, J., 2004. Sum capacity of Gaussian vector broadcast channels. Information Theory, IEEE Transactions on, 50(9), 1875-1892.

Weingarten, H., Steinberg, Y. \& Shamai, S., 2006. The Capacity Region of the Gaussian Multiple-Input Multiple-Output Broadcast Channel. Information Theory, IEEE Transactions on, 52(9), 3936-3964.

\section{Appendix}

\subsection{DCAP derivation}

For the co-located transmit antennas case $\overline{\mathbf{H}}_{i} \overline{\mathbf{H}}_{i}^{H},(i=M, M+1)$, is $\tilde{\mathcal{W}}_{K N}(i, \boldsymbol{\Xi})$ distributed. But since $\overline{\mathbf{H}}_{i} \overline{\mathbf{H}}_{i}^{H} \sim \boldsymbol{\Xi}^{1 / 2} \mathbf{A}_{i} \boldsymbol{\Xi}^{1 / 2}$, where $\mathbf{A}_{i}$ is $\tilde{\mathcal{W}}_{K N}\left(i, \mathbf{I}_{K N}\right)$ distributed, the DCAP can be expressed by:

$$
\begin{aligned}
\Delta C_{M, K, N}^{M+1, K} & =\mathbb{E}\left[\log \left|\overline{\mathbf{H}}_{M+1} \overline{\mathbf{H}}_{M+1}^{H}\right|\right]-\mathbb{E}\left[\log \left|\overline{\mathbf{H}}_{M} \overline{\mathbf{H}}_{M}^{H}\right|\right] \\
& =\mathbb{E}\left[\log \left|\mathbf{A}_{M+1}\right|\right]-\mathbb{E}\left[\log \left|\mathbf{A}_{M}\right|\right] \\
& =\sum_{l=0}^{K N-1} \Psi(M+1-l)+\sum_{l=0}^{K N-1} \Psi(M-l) \\
& =\sum_{n=M-K N+1}^{M} \frac{1}{n} \approx \log \left(1+\frac{K N}{M-K N+\beta}\right)
\end{aligned}
$$


where $\Psi(l)=-\gamma+\sum_{n=1}^{l-1}$, is the Euler's digamma function. The result from the second line comes from the determinant property $(|\mathbf{A B}|=|\mathbf{A}||\mathbf{B}|)$ and the result from the third line from lemma 1 of (Juyul Lee \& Jindal 2007) $\left(\mathbb{E}\left[\log \left|\mathbf{A}_{i}\right|\right]=\sum_{l=0}^{i-1} \Psi(i-l)\right.$ ). To obtain an approximation to the partial sum of the harmonic series we have used $\sum_{n=1}^{M} 1 / n=\gamma+\log (M+\beta)$ which has a maximum error of $4.1 \times 10^{-3}$.

9.1 Capacity Increase by the connection of one more user, for $N=1$ and $M=1$

For $N=1$ and $M=1$ the broadcast channel ergodic sum-capacity, equation (7), in the high SNR regime, simplifies to:

$$
\overline{\mathbf{C}}_{D P C}^{1, K, 1}=\mathbb{E}\left[\log \left(P \max _{k=1 \ldots K}\left(\xi_{k}^{1 / 2} h_{k}^{H} h_{k} \xi_{k}^{1 / 2}\right)\right)\right]=\mathbb{E}[\log (P x)]
$$

where $\xi_{k}$ is the average SNR of user $k, h_{k} \sim \tilde{\mathcal{N}}(0,1)$ and $x=\max _{k}\left(\xi_{k} h_{k}^{H} h_{k}\right)$. It is easy to show that $d \Delta C_{1, K, 1}^{1, K+1,1} / d \xi_{k} \leq 0$ for $k \neq K+1$ and that the opposite happens for $k=K+1$. Thus the maximum of $\Delta C_{1, K, 1}^{1, K+1,1}$ is obtained when $\xi_{k}=\xi$ for all users, if the new connected user is always the one which implies the highest increase in the ergodic sum-capacity. It can also be proven that the same value for $\Delta C_{1, K, 1}^{1, K+1,1}$ is obtained for any $\xi$. Hence, for simplicity we consider that $\xi=1$. Thus $x$ has cumulative distribution function $F_{X}(x, K)=F_{Y}(y)^{K}$, (Liu \& Li 2005b), where $y \sim \xi_{k} h_{k}^{H} h_{k}$ is exponential distributed with mean 1 . Thus the capacity increase by the connection of an additional user to the system can be expressed by ${ }^{7}$ :

$$
\Delta C_{1, K, 1}^{1, K+1,1}=\int_{0}^{\infty} \log (x) d F_{X}(x, K+1)-\int_{0}^{\infty} \log (x) d F_{X}(x, K)
$$

which after the integral evaluation gives the result shown in equation (26).

7 The $P$ term cancel out from the expression since it appears in $\overline{\mathbf{C}}_{D P C}^{1, K+1,1}$ and $\overline{\mathbf{C}}_{D P C}^{1, K, 1}$. 


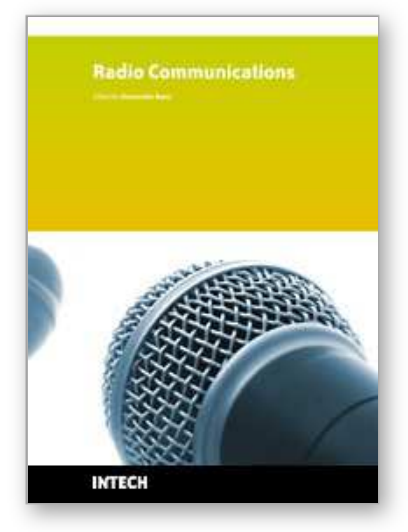

\author{
Radio Communications \\ Edited by Alessandro Bazzi
}

ISBN 978-953-307-091-9

Hard cover, 712 pages

Publisher InTech

Published online 01, April, 2010

Published in print edition April, 2010

In the last decades the restless evolution of information and communication technologies (ICT) brought to a deep transformation of our habits. The growth of the Internet and the advances in hardware and software implementations modified our way to communicate and to share information. In this book, an overview of the major issues faced today by researchers in the field of radio communications is given through 35 high quality chapters written by specialists working in universities and research centers all over the world. Various aspects will be deeply discussed: channel modeling, beamforming, multiple antennas, cooperative networks, opportunistic scheduling, advanced admission control, handover management, systems performance assessment, routing issues in mobility conditions, localization, web security. Advanced techniques for the radio resource management will be discussed both in single and multiple radio technologies; either in infrastructure, mesh or ad hoc networks.

\title{
How to reference
}

In order to correctly reference this scholarly work, feel free to copy and paste the following:

Daniel Castanheira and Atilio Gameiro (2010). Single/Multi-User MIMO Differential Capacity, Radio Communications, Alessandro Bazzi (Ed.), ISBN: 978-953-307-091-9, InTech, Available from: http://www.intechopen.com/books/radio-communications/single-multi-user-mimo-differential-capacity

\section{INTECH}

open science | open minds

\author{
InTech Europe \\ University Campus STeP Ri \\ Slavka Krautzeka 83/A \\ 51000 Rijeka, Croatia \\ Phone: +385 (51) 770447 \\ Fax: +385 (51) 686166 \\ www.intechopen.com
}

\author{
InTech China \\ Unit 405, Office Block, Hotel Equatorial Shanghai \\ No.65, Yan An Road (West), Shanghai, 200040, China \\ 中国上海市延安西路65号上海国际贵都大饭店办公楼 405 单元 \\ Phone: +86-21-62489820 \\ Fax: +86-21-62489821
}


(C) 2010 The Author(s). Licensee IntechOpen. This chapter is distributed under the terms of the Creative Commons Attribution-NonCommercialShareAlike-3.0 License, which permits use, distribution and reproduction for non-commercial purposes, provided the original is properly cited and derivative works building on this content are distributed under the same license. 